\title{
FIRST POSTER DISCUSSION
}

PILACHOWSKI: Welcome to our first poster discussion session on the topic of globular clusters in the Galaxy. I'd like to begin the discussion with the paper by Sweigart, Greggio, and Renzini. These authors have constructed new evolutionary sequences for low and intermediate mass stars evolving from the main sequence to the onset of core helium ignition in order to map the transition from intermediate mass stars without a prominent red giant phase to low mass stars which do undergo a prominent red giant phase. Their results show the RGB phase transition begins at an age of 500 to 600 million years, depending on composition. How well determined is this transition age from observations of star clusters? Do any star clusters with prominent red giant branches have ages less than a billion years?

\section{WALLERSTEIN: M 11}

PILACHOWSKI: It's young, all right, but it doesn't have much of a red giant branch above the clump. I can contrast two clusters, NGC 7789 and NGC 752, both of which have ages of about 1.5 Gyr. NGC 7789 has a very nice red giant branch; Saha and I have been searching for red giants in NGC 752, but we can find no stars above the luminosity of the clump.

RENZINI: The best observational test will come in the Magellanic Clouds, but we need more data for the clusters.

PILACHOWSKI: The paper by McClure, Stetson, Hesser, Smith, and VandenBerg presents luminosity functions for several clusters with a range of metallicity, and finds a trend between the power law exponent of the main sequence mass function and metallicity. King has added one additional paper to the discussion which suggests that mass functions determined from the outer parts of clusters are probably reliable - so there is no need to get all the way into the center. The determination of luminosity functions is a lot of work, but we really need to see more of them determined!

BELL: I'd like to emphasize that there are really two problems in determining luminosity functions; we need a better defined system than $B$ and $V$, and we need broad bands to get to the faintest stars.

COHEN: Please tell us the magnitude of the crowding corrections applied to the observed star counts so we can decide how much credence to place on these results.

MCCLURE: As I remember the corrections are less than about 308 for the data appearing in the figures of this paper.

DA COSTA: Dynamical Evolution - the loss of low mass stars via tidal stripping, escape, etc - can mean that current mass functions are not necessarily related to INITIAL mass functions. For example, NGC 6397 
is a very metal poor cluster with a current mass function that is deficient in low mass stars (alpha - 0, Da Costa 1982). But this is a very dynamically evolved cluster that may have had an initial mass function that supports the proposed correlation.

KING: It seems to me that the existence of a correlation between mass function and metallicity, undisturbed by dynamical properties, implies that there has not been any serious loss of low-mass stars.

PRYOR: R. McClure, G. Smith and myself have calculated mass segregation corrections for luminosity function power law exponents. Our results are similar to those in King's poster. The exponents become smaller by $\Delta \mathrm{X} \sim 1$, but the trend with metallicity is preserved. More theoretical work is needed, though, on the amount of mass segregation expected at the very large radii where some of the luminosity functions are measured.

PILACHOWSKI: Let's move on to a discussion of the papers on the subject of cluster structure parameters. The first of these papers, by Peterson and Reed, offers new determinations of the core and tidal radii, and central relaxation times, densities, velocity dispersions, and escape velocities, among other parameters. The authors urge the use of these new values, which have corrected systematic errors in earlier results. The paper, by White and Shawl, tabulates axial ratios and major-axis orientations for 104 galactic globular clusters, and suggests that galactic tidal effects don't elongate clusters. I also recommend a peek at Figure 5 of their paper for a most interesting suggestion! People who want to correlate various composition parameters like $\mathrm{CN}$ with cluster flattening, etc. should probably look at these new data.

NORRIS: For the dependence of degree of CN strengthening on flattening I incorporate the data of White and Shawl!

PILACHOWSKI: Now we come to three papers which are dear to my heart! The papers by Leep, Wallerstein, and Oke; Gratton, Quarta, and Ortolani; and Pilachowsksi and Sneden; all present the results of detailed analyses of high resolution CCD spectra of globular cluster giants, and M 71 giants in particular. All three papers follow similar techniques, and all three incorporate Arcturus as a standard. I believe that all the authors of these papers will agree with me that the introduction of new spectrographs, and particularly new detectors, in the last 7 years has provided a tremendous gain in our ability to obtain high-quality spectra of faint stars in globular clusters. We owe a great debt to the engineers who have made this possible. How nice it is that all these papers agree that the iron abundance in $M 71$ is near -0.75 ! The controversy can be laid to rest.

TRIMBLE: Your abstract doesn't mention the amount - what is the $[\mathrm{Fe} / \mathrm{H}]$ of $\mathrm{M} 71$ ? 
PILACHOWSKI: -0.75 for 2 stars.

BELL: Is TiO contamination a possible resolution of the low echelle abundances? Indeed, aren't there Tio bands all over to worry about?

PILACHOWSKI: The stars anabyzed here are too hot to form much TiO, and we see no evidence for it in the spectrum.

GEISLER: In the case of the cooler 47 Tuc giants in particular, there are regions where the Tio bands are so strong that they lower the "continuum" and can cause abundance underestimates by -0.2 dex. In relative clean regions one does still need to worry about individual lines blending with Tio features.

PILACHOWSKI: Of course, we are careful to select lines that appear clean and unblended in the Arcturus atlas! It is also true that the line list used in the earlier analysis of cool 47 Tuc giants contained few lines in the regions Doug has shown to be contaminated with Tio.

PILACHOWSKI: I'd like to ask Rafaele Gratton what the prospects are for determining the abundance of oxygen in his 47 Tuc giants.

GRATTON: The geocentric radial velocity at the observing time was too low; therefore, the stellar line was blended with telluric emission. We could not derive reliable abundances.

PILACHOWSKI: The next paper by Rose and Tripicco reports on the integrated spectra of metal-rich galactic globular clusters. They find that the relative amounts of light contributed by dwarfs and giants varies from one cluster to another. Do you have any further results to add?

ROSE: We have very recently obtained integrated spectra of the core of 47 Tuc. Both its Sr II/Fe I and 3888/3859 indices are high, from which we infer that the core is dominated by dwarf light at $4000 \AA$, and that the mean core $C N$ anomaly is very large. This result probably rules out the possibility that the observed spread in the $\mathrm{Sr}$ II/Fe I index between clusters is due to an age spread.

SCHOMMER: Which is your other cluster (beside 47 Tuc) which has a well-determined age and dwarf/giant ratio? I'm not sure any age is really reliable to 1 Gyr.

ROSE: Of the two clusters with well determined ages, only 47 Tuc shows an excess of dwarfs. The other one is $M 71$, and it does not show an excess of dwarfs.

KING: If you put an excess of dwarfs in the center of a well-relaxed cluster, you make life quite difficult for those of us who try to model the clusters dynamically. 
COHEN: As I recall, the integrated light measurements of $C O$ band strengths in globular clusters show no indication of dwarfs dominating the light. Are the samples disjoint or do I recollect incorrectly or are the $\mathrm{CO}$ measurements irrelevant?

ROSE: A factor of 2 variation in the relative amounts of dwarfs' vs. giants' light at $4000 \AA$, which is what we infer from the strontium index, will not necessarily show up at 2.2 microns, where the giant light will dominate except in extreme circumstances.

PILACHOWSKI: The paper by Altner discusses two mysterious broad absorption features at 1400 and $1600 \AA$ in globular clusters. Bruce, would you like to tell us what you suspect the origin of these features might be?

ALTNER: There are 3 possibilities: the bands could be artifacts of the camera, the features could be real in individual stars, and then show up in the integrated spectra, or the short wavelength tails of the light of cooler stars may contribute a "saddle" effect.

PILACHOWSKI: Smith reports on $\mathrm{V}$ and 1.0 micron photometry of giants in $\omega$ Cen. He finds a broad giant branch even in the $(V,(V-1 \mu))$ diagram. Graeme, would you like to comment further on this paper?

SMITH, G.: (V - $1 \mu)$ photometry was done of $\omega$ Cen giants, because John Norris and I wanted to obtain a color from which we could measure effective temperatures of stars for which no infrared photometry was available. We used ( $\mathrm{V}$ and $1 \mu$ ) in an attempt to obtain a long baseline and avoid the problem with (R-I), which does not show a large spread on the $w$ Cen giant branch.

PILACHOWSKI: Now we come to the paper by Armandroff. I was impressed by the tight correlation between the calcium infrared triplet strength and $[\mathrm{Fe} / \mathrm{H}]$. Will you be applying this technique to more systems, reddened clusters in the direction of the galactic center? Radial velocities and metallicities are being derived from these spectra. It may be possible to apply this technique to extragalactic globulars. A parallel technique is being developed for clusters which are too diffuse for integrated light spectroscopy using individual-giant spectroscopy, again at the calcium triplet.

PETERSON: Are you planning to include galactic bulge giants in this program? These are basic ingredients for metal-rich extragalactic objects.

ARMANDROFF: It would be useful to include those giants.

NORRIS: Are you finding clusters with roughly solar abundance, similar to the most metal-rich objects found by Zinn among his disk globular cluster group? 
DA Costa: For very cool, luminous giants the triplet line strengths tend to saturate for abundances greater than $[M / H]-1.0 \mathrm{dex}$, so metal abundance discrimination at high abundances in individual stars is reduced.

COHEN: There are some problems in extending the use of the IR calcium triplet to extragalactic globular clusters - you are limited by the atmospheric emission lines.

HESSER: Hugh Harris, Gretchen Harris, and I successfully obtained radial velocities for NGC 5128 globular clusters using the IR calcium triplet for clusters having $\mathrm{V}<-19$. We haven't yet tried to calculate abundance indices because, in part, we were not impressed by the difference in line strengths in the few giants we observed in clusters of widely differing metallicity.

PILACHOWSKI: Lee, Demarque, and Zinn have found that they can produce bimodal distributions of horizontal branch stars with a monomodal distribution of initial parameters. In your models, do you find it easier to create horizontal branch gaps at some effective temperatures than at others?

LEE: The positions of gaps depend on the $[\mathrm{Fe} / \mathrm{H}]$ of the clusters. The combination of the shapes of the evolutionary tracks and the evolutionary speed along each track can naturally produce an apparent bimodal distribution of the horizontal branch, or gaps.

NORRIS: Would you predict that all horizontal branches which span the RR Lyrae gap should have bimodal distributions?

LEE: The horizontal-branch morphology strongly depends on the mean horizontal branch mass and dispersion factor.

PILACHOWSKI: Now we have two papers on horizontal-branch stars by Rood and Crocker, and Crocker and Rood. They find differences in surface gravity among very blue horizontal-branch stars which may be due to differences in the helium abundance or to rotation. In the second paper they detect a strong HE I 4026 line in two hot horizontal-branch stars in NGC 6752. The presence of strong helium lines may give us the opportunity of determining helium abundances in horizontal branch stars.

NORRIS: Given that one seems a range of helium abundance among blue horizontal-branch stars, how does one know which objects are telling you the truth about the Population II helium abundance?

CROCKER: It's important to observe a large enough sample of stars.

PILACHOWSKI: There are two papers by Philip and Samus and by Philip which discuss spectra and four-color photometry of blue horizontal-branch stars in several globular clusters. They find 
evidence for some stars with stronger calcium lines, and some stars with high Strömgren $c_{1}$ indices. They need more data to confirm that these two groups of stars are the same.

SMITH, H,: Do you see unusual abundances of any element but calcium? In particular, can you see anything unusual about lines which might be sensitive to diffusion?

PHILIP: The dispersion of these spectra is such that the K-line can be seen and measured. Weaker lines can not be resolved, and thus $I$ have no information on other lines. It would take spectra at a higher dispersion to resolve such lines.

PILACHOWSKI: Turning to the question of the similarity of the cluster and field halo populations, we have two papers by Laird, Carney, Latham, and Kurucz and by Ratnatunga. The first of these examines the metallicity distribution function of halo dwarfs determined from low signal-to-noise, high resolution spectra using cross-correlation techniques. They find no compelling evidence that the metallicity distribution functions for the field stars and for the clusters are different.

LAIRD: I would like to clarify our result regarding the metallicity distribution functions of the field dwarfs and globular clusters. By a Kolmogorov-Smirnov test, which is a very conservative test, one cannot be sure that their distributions are different, but they certainly look different. The lack of certainty is largely due to the small number of objects.

NORRIS: What was the level of significance at which the cluster and field star distributions were different.

ZINN: The difference between the observed metallicity distribution of the clusters and the simple model may not be significant. The model predicts that only a very few additional metal poor clusters should exist, and it may be nothing more than small number statistics that these clusters are not in the present sample, which, by the way, is not a complete sample of all of the globular clusters in the Milky Way.

ZINNECKER: The result that halo field stars can be more metal-poor than the cluster stars may contradict the prediction of the theory of Fall and Rees (1985), the halo field stars should form only after the metallicity in the proto-galaxy is raised to $0.1 \mathrm{Z}_{\mathrm{o}}$, or thereabouts.

ZINN: If my memory is correct, the work of Fall and Rees suggested that individual field stars could form only when the metal abundance of the halo was moderately high (i.e. [Fe/H] -1.0). According to their picture, it is possible to form more metal-poor clusters of stars, and I believe that they suggest that the metal-poor halo stars originated in clusters that were disrupted. 
PILACHOWSKI: Ratnatunga finds evidence for a thick disk in a sample of 58 giants located by his objective prism survey. His thick disk-1ike component is rotating with the disk, and has a velocity dispersion of $50 \mathrm{~km} / \mathrm{sec}$.

PILACHOWSKI: The next group of papers we will discuss concern the galaxy cluster system as a whole. The paper by Colin, suggests that the cluster system is in prograde rotation, and identifies groups of clusters with distinct rotation. The second paper by Tucholke, Brosche, and Geffert presents their efforts to determine absolute proper motions for clusters referred to extragalactic sources. Cudworth determines an astrometric distance to $M 22$, and Djorgovski describes his search of the IRAS data base to locate hidden globular clusters. I'd like to begin the discussion of these papers by asking Kyle how seriously we should take his new distance to M 22 .

CUDWORTH: We want to move M 22 closer by $\sim 0.5$ magnitudes, but some of this is likely to be due to reddening problems in this cluster. Other papers at this meeting will claim fainter RR Lyrae absolute magnitudes, so there may not be a big discrepancy. We also do not currently understand M 22 dynamics well enough.

SCHOMMER: I have a question for Bob Zinn. Which is the outer halo blue horizontal-branch cluster without a known $[\mathrm{Fe} / \mathrm{H}]$ ?

ZINN: The cluster is Pal 15.

PILACHOWSKI: Finally, we have a paper dealing with the interstellar medium. Straizys and Janulis describe their study of the reddening toward M 71, M 56, and NGC 6712. They note that the reddening towards $M$ 71 varies by a factor of 3 across the cluster. Zdanavicius also presents a paper on the use of the Vilnius photometric system to separate cluster and field stars.

WEBBINK: In compiling color excesses of globular clusters for the appendix to IAU Symposium 113, I was struck by the fact that in virtually every case of appreciable reddening $(E(B-V)>0.2)$, variations in reddening over the face of the cluster in question are quite apparent visually on blue sky survey plates. It is somewhat misleading to assign a single value to the reddening of these clusters.

PETERSON: Reddening of M 4 within the narrow area studied by Richer and Fahlman must be very constant to reproduce the narrow width of the main sequence turnoff.

RICHER: Although one field in $M 4$ with an area of $3^{\prime} \times 5^{\prime}$ shows a tight turnoff, at least one other shows differential reddening of the indices of $0.03-0.05$ in $E(B-V)$.

PILACHOWSKI: This concludes the discussion. I'd like to thank the 
authors of all the papers in this session, and all of you for a stimulating discussion. 